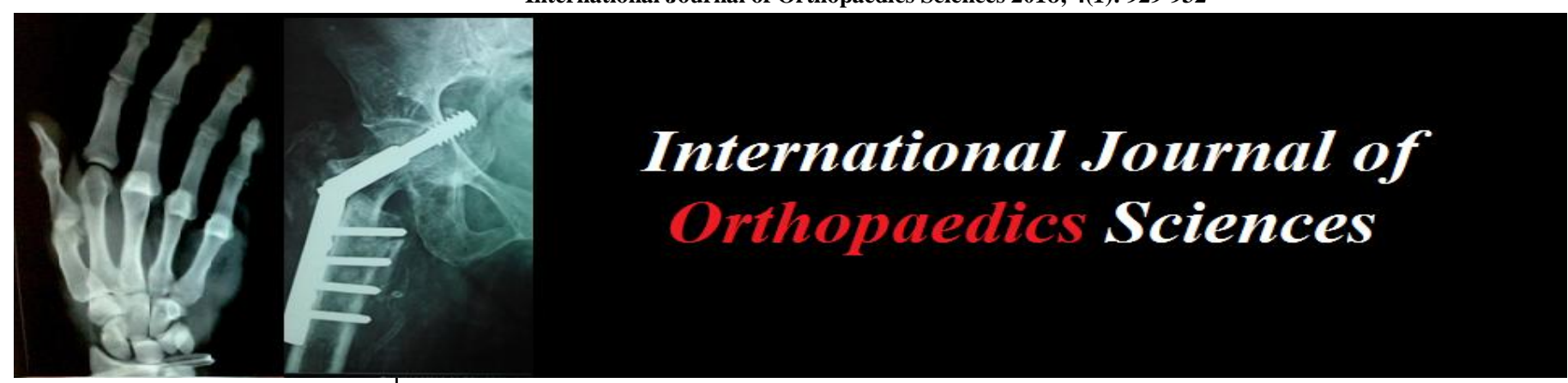

ISSN: $2395-1958$

IJOS 2018; 4(1): 929-932

(C) 2018 IJOS

www.orthopaper.com

Received: 03-11-2017

Accepted: 04-12-2017

Satya Kumar Koduru

Professor of Orthopaedics, Nri

Academy of Sciences,

Mangalagiri, Guntur, Andhra

Pradesh, India

\section{Avinash D}

Junior, Resident, Department Of

Orthopaedics, Nri Academyof

Sciences, Mangalagiri, Guntur,

Andhra Pradesh, India
Correspondence

Satya Kumar Koduru

Professor of Orthopaedics, Nri

Academy of Sciences,

Mangalagiri, Guntur, Andhra

Pradesh, India

\section{Different surgical methods to manage avascular necrosis of head of femur}

\section{Satya Kumar Koduru and Avinash D}

DOI: https://doi.org/10.22271/ortho.2018.v4.i1n.132

\section{Abstract}

A prospective study was carried out on 30 patients who underwent core decompression, bipolar hemiarthroplasty, cemented/uncemented Total Hip Replacement in Department of Orthopaedics, NRI Institute of Medical Sciences. This study has shown that, the mean age of patients in the study group was $42.30 \pm 11.43$ years. About $43.3 \%$ of the avascular necrosis patients in the study group belonged to $31-40$ years age group. The sex distribution of the study group has shown that, about $76.7 \%$ of the patients were males and $23.3 \%$ were females. The mean ROM score of study group during preoperative stage was $2.40 \pm 1.25$ and postoperative stage was $4.63 \pm 0.49$. The difference between the ROM scores during pre and postoperative stage was statistically significant. The type of implant used in eth study group. Stryker implant was used in $73.3 \%$ and Link implant was used in $26.7 \%$ of the study group. In the study group, most (93.3\%) of the patients had no complications. The common complication in the study group was foot drop in 2 patients which is $6.7 \%$. Analysis shows the complications of total hip replacement according to type of implants used in the study group. Most (93.3\%) of the patients in the study group had no complications. But for 1 patient used link implant had foot drop and for 1 patient used stryker implant had foot drop. The outcome after total hip replacement was excellent in $80.0 \%$ of the study group. About $20.0 \%$ of the study group had good outcome.

Keywords: surgical, manage, necrosis, head of femur

\section{Introduction}

Methodology

A prospective study was conducted on patients having avascular necrosis of femoral head, who came to department of Orthopaedics and referred from other departments from july 2014 to November 2016 in NRI Institute Of Medical Sciences And General Hospital, chinkakani, Guntur, Andhra Pradesh. The clearance from institutional ethical committee was obtained before starting the study.

A total of 30 patients, who had given an informed, bilingual and written consent, posted for surgery were included in to the study. Patients were admitted and examined according to protocol both clinically and radiologically, and functional outcome was assessed by distribution of - Harris hip scorell both preoperatively and postoperatively and the patients are reviewed with post op x-rays immediately after surgery at the end of $6,12,24$ weeks after the surgery.

\section{The inclusion and exclusion criteria were as follows: \\ Inclusion criteria}

Patients of avascular necrosis of femoral head.

Patients in the age group of 30 to 75 years.

Patients willing to give informed consent.

\section{Exclusion criteria}

Patients who are not medically fit for surgery

Patients with age less than 30 years.

Patients not willing for surgery.

On admission to the ward, a detailed history of the patients was taken. This included age, sex, 
occupation, complaints, associated medical illness. Following this, they were subjected to a thorough clinical examination and general condition was assessed and accordingly corrective measures were taken to correct the general being of the patients.

Routine blood investigations were done for all the patients. Special attention was paid to CRP and ESR and if these were abnormal, surgery was deferred. Standard antero-posterior and lateral X-rays were taken including pelvis with both hips. Analgesics, antibiotics, tetanus toxoid and blood transfusions were given as needed before surgery.

\section{Preoperative assessment}

The physical fitness of the patient undergoing a major surgery was assessed. Physical examination included examination of spine and both lower extremities including opposite hip, both knees and foot. Trendelenburg test to assess the abductor musculature mechanism was done.

The patients were evaluated using the modified Harris hip score. The score taken into accont pain, function, range of movements and deformities. Also a mention of the limb length discrepancy and flexion contracture is made Neurovascular status of affected extremity was evaluated. Any occult infections like skin lesions, dental caries and urinary tract infections were identified and treated preoperatively.

The aim of the pre-op planning was to obtain the following results post operatively An acetabular socket located in the anatomical position.

\section{Restoration of limb length. \\ Restoration of abductor arm}

Radiograph of the pelvis with both hips with proximal half of shaft of femur APview was taken for all patients. The radiograph was evaluated for:-Centre of rotation of femoral head located in its normal anatomical position

\section{Preparation}

\section{Taken an informed consent}

Shaved the extremity, perineal area, hemipelvis to atleast $10 \mathrm{~cm}$ proximal to theiliac crest and wash with soap as soon as possible before surgery and cover with sterile towels. Prophylactic antibiotics: A combination of piperacillin and tazobactum was administered the night and morning before surgery and was continued postoperatively for 3 days.

Templating was done for the aectabular and femur components. The appropriate acetabular cup size, and anteversion was determined.

\section{Results}

The present study consists of 30 cases of of avascular necrosis of head of femur treated at N R I General Hospital, Chinnakakani, treated between July 2014 to November 2016. 4 cases were belong to stage-I of ficat and arlet classification. 6 cases were belong to stage II of ficat and arlet classification. 10 cases were belong to stage-III of ficat and arlet classification. 10 cases were belong to stage-IV of ficat and arlet classification.The following are the observations made to the available data analysed as follows

\section{Evaluation of Results}

The evaluation of results was done by using the new method reported by W.H. Harris, 1969. The rationale of this new method of evaluation is as described. Pain and functional capacity are the two basic considerations. They constitute the indications for surgery in the vast majority of patients with hip problems, and hence receive heaviest weightings. In specific cases, correction of deformity or restoration of motion may be of prime importance but such cases are uncommon. Based on this reasoning a point scale with a maximum of 100 points is used with the following maximum possible scores.

\section{Discussion}

Avascular necrosis (also osteonecrosis, bone infarction, aseptic necrosis, ischemic bone necrosis, and AVN) is a disease where there is cellular death (necrosis) of bone components due to interruption of the blood supply. Without blood, the bone tissue dies and the bone collapses There are many theories about what causes of AVN, includes chemotherapy, alcoholism, excessive steroid usage, caisson disease (decompression sickness), vascular compression, hypertension, vasculitis, arterial embolism and thrombosis, damage from radiation, bisphosphonates (particularly the mandible), sickle cell anemia and Gaucher's Disease,. In some cases it is idiopathic (no cause is found). Rheumatoid arthritis and lupus are also common causes of AVN. Prolonged, repeated exposure to high pressures (as experienced by commercial and military divers) has been linked to AVN, though the relationship is not well-understood.

While it can affect any bone, about half of cases show multiple sites of damage, avascular necrosis primarily affects the joints at the shoulder, hip. The classical sites are: head of femur, neck of talus and waist of scaphoid. Clinical avascular necrosis most commonly affects the ends (epiphysis) of long bones such as the femur (the bone extending from the knee joint to the hip joint). Other common sites include the humerus, shoulders, ankles and the jaw.

The disease may affect just one bone, more than one bone at the same time, or more than one bone at different times. Avascular necrosis usually affects people between 30 and 50 years of age; about 10,000 to 20,000 people develop avascular necrosis of the head of the femur in the US each year.

A variety of methods are now used to treat avascular necrosis, the most common being the total hip replacement or THR. Total hip arthroplasty is a well-documented surgical procedure. It relieves pain and functional disability experienced by patients with moderate to severe arthritis of the hip, secondary to AVN and improving their quality of life A prospective study was carried out on 30 patients of which ten patients underwent core decompression, ten patients underwent bipolar arthroplasty and remaining ten underwent cemented/uncemented Total Hip Replacement.

\section{Results}

\section{Age and Sex}

This study has shown that, the mean age of patients in the study group was $42.30 \pm 11.43$ years. About $43.3 \%$ of the avascular necrosis patients in the study group belonged to 31 40 years age group. In contrary to these findings, a multivariate analysis identified young age at onset of avascular necrosis.

The sex distribution of the study group has shown that, about about $76.7 \%$ of the patients were Males and $23.3 \%$ were females. Other studies like Tofferi JK, Gilliland W, also found the same results. 


\section{Etiology}

The analysis of patients for the etiology of AVN showed that $70.0 \%$ of the patient developed AVN of hip joint without any known cause (idiopathic), $16.7 \%$ of patients developed AVN secondary to corticosteroid, and secondary to post trauma $13.3 \%$ patients developed AVN of the hip joint. in a study by Koo KH, Kim R, Kim YS, et al. showed, $65 \%$ of AVN due to idiopathic cause and $10 \%$ to $30 \%$ cases due to corticosteroid therapy which is quite similar to this study.

\section{Core decompression}

Core decompression with cancellous bone grafting is a safe and effective procedure for the treatment of early AVN of the femoral head before collapse of femoral head. Results with this form of treatment are considerably better than those obtained in patients treated nonoperatively. The results in hips with early (stages I and II) AVN were only slightly better than those of hips with advanced (stages III and IVA) disease.

\section{Type of arthroplasty}

Bipolar hemiarthroplasty is done in stage 3 of ficat arlet staging wih out acetabular involvement and total hip replacement in stage 4.Majority of the patients $(86.7 \%)$ had uncemented type of arthroplasty and $13.3 \%$ had undergone hybrid type of arthroplasty. The new generation of uncemented prosthesis had demonstrated improvement in clinical and radiological outcomes compared with those associated with early designs of prosthesis inserted without cement.

\section{Pain relief}

The mean pain score during preoperative period was $10.13 \pm 1.96$ and during postoperative stage was $42.13 \pm 2.03$. Sochart et al. observed that all the surviving patients had substantial pain relief and improvement of function. Pain relief is more in total hip replacement than bipolar hemiarthroplasty and coredecompression.

\section{Functional gait \& activity}

The mean functional gait score during pre-operative stage was $10.53 \pm 6.96$ and during postoperative stage was $30.33 \pm 2.31$. The mean functional activity score during preoperative stage was5.17 \pm 1.62 and during post-operative stage was $11.40 \pm 1.07$ In a study by Katz JN et al, ambulation and functional ability improved in most of the patients

\section{Range of movement score}

The mean ROM score of study group during pre-operative stage was $2.40 \pm 1.25$ and postoperative stage was $4.63 \pm 0.49$. The difference between the ROM scores during pre and postoperative stage was statistically significant. In a study by Katz JN, Phillips CB, the improvement in sum total range of motion was achieved in patients with avascular necrosis of femoral head and preoperative sum total range of motion was maintained or improved in hips with a painful arc of motion. They have also noted that most of the limited motion in patients with avascular necrosis is due to idiopathic

\section{Complications of THR}

Most (93.3\%) of the patients in the study group had no complications. The common complication in the study group was Foot drop in 2 patients which is $6.7 \%$ In a study by Meek RM, Garbuz DS, intraoperative fracture was observed in $4.3 \%$ of hips, sciatic nerve palsy was observed in $1.1 \%, 14 \%$ of the cases were revised because of aseptic loosening. in a study by
Learmonth ID101 showed periprosthetic fracture in $8.6 \%$ cases

\section{Limb shortening}

In the study group, postoperative limbshortening was not seen in $93.3 \%$ of the study group. About $6.7 \%$ each of the study group had limb shortening of $1 \mathrm{~cm}$ and $1.5 \mathrm{cms}$. Over lengthening is more common than a residually shortened leg and a lengthened limb is more poorly tolerated. Konyves and Bannister ${ }^{117}$ noted that lengthened limbs were also associated with lower clinical hip scores. Limb-length discrepancy can result from a poor preoperative patient evaluation as well as intraoperative technical errors with regard to the level of resection of the femoral neck, the prosthetic neck length, or the failure to restore offset.

\section{Conclusion}

Avascular necrosis (AVN) primarily affects the axial skeleton and hip joints. The other joints such as humerus, shoulders, ankles and the jaw also can get involved. Patients with avascular necrosis are usually young and more active and problems are related to function, men are usually affected more than women. Idiopathic osteonecrosis in more common followed by steroid induced and post-traumatic AVN. Total hip arthroplasty is a well-documented surgical procedure. It relieves pain andfunctional disability experienced by patients with moderate to severe arthritis of the hip, secondary to AVN and improving their quality of life. The outcome of the total hip replacement in AVN of hip joint is determined bymany factors including the design of component, the selection of the patients, and the operative technique. The results of the procedure needs long term studies for evaluating the complete effect. His study suggests that the current generation of uncemented implants used intotal hip replacement for arthritis of the hip joint secondary to AVN, Bipolar hemiarthroplasty and core decompression provides satisfactory clinical and radiographic outcomes after an intermediate duration follow up.

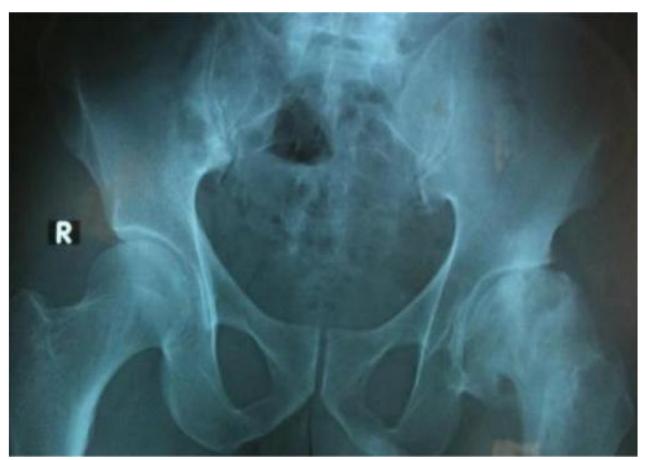

Fig 1: showing arthritic changes in left hip

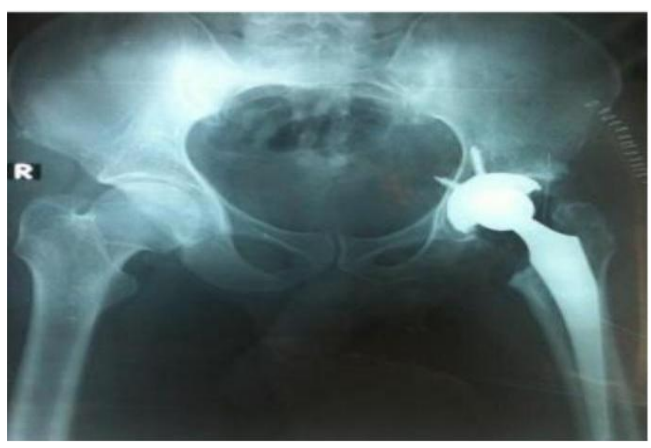

Fig 2: post-operative x ray of total hip replacement 

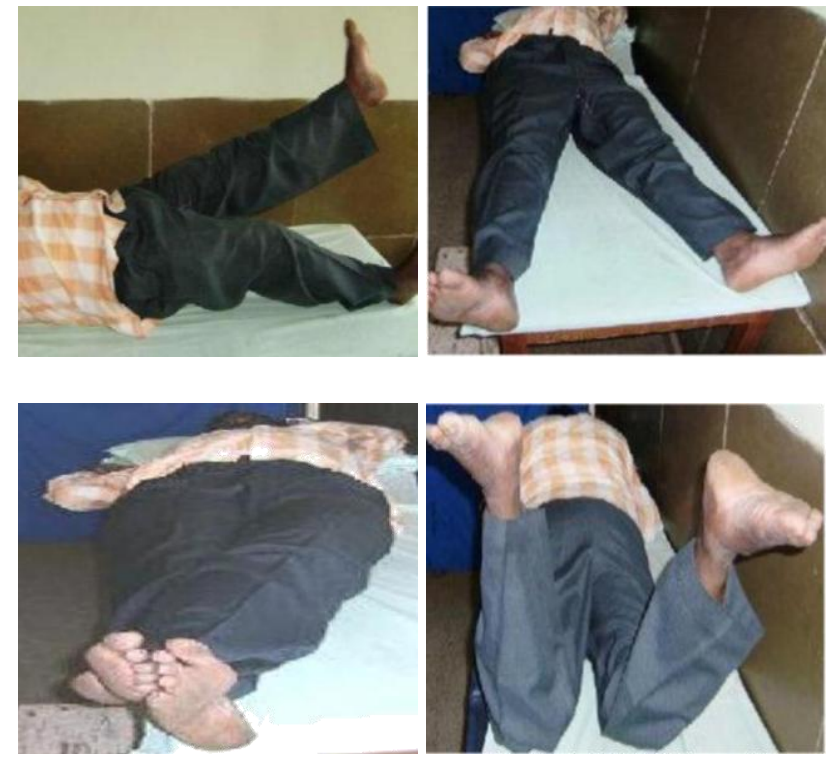

Fig 3: showing hip range of movements

\section{References}

1. Siopack JS, Jergesen HE. Total hip arthroplasty. West J Med. 1995; 162:243-249.

2. Title: Campbell's operative orthopaedics, chapter 7,Arthroplasty of hip, James W. Harkes, John R. Crockarell $11^{\text {th }}$ edn, 335-3.

3. Arden N, Nevitt MC. Osteoarthritis: epidemiology. Best Pract Res Clin Rheumatol. 2006; 20:3-25

4. Felson DT. An update on the pathogenesis and epidemiology Of osteoarthritis. Radiol Clin North Am. 2004; 42:1-9.

5. Zhang Y, Jordan JM. Epidemiology of osteoarthritis. Rheum Dis Clin North Am. 2008; 34:515-29.

6. Signe Rosenlund, Leif Broeng, Carsten Jensen, Anders Holsgaard-Larsen. The effect of posterior and lateral approach on patient-reported outcome measures and physical function in patients with osteoarthritis, undergoingtotal hip replacement: a randomised controlled trial protocol. Rosenlund et al. BMC Musculoskeletal Disorders. 2014; 15:354

7. Carolina Santanna Umpierres, Tiango Aguiar Ribeiro, Ângela Elisabete, Marchisio. Rehabilitation following total hip arthroplasty evaluation over short follow-up time: Randomized clinical trial. JRRD. 2014; 51(10):1567-1578.

8. Steinberg ME. Diagnostic imaging and role of stage and lesion size indetermining outcome in osteonecrosis of the femoral head. Tech Orthop. 2001; 16:6-15.

9. Khanuja HS, Mont MA, Etienne G, Hungerford DS. Treatment algorithm forosteonecrosis of the hip. Techn Orthop. 2001; 16:80-9.

10. Steinberg, Marvin E. Osteonecrosis: Merck Manual of Diagnosis and Therapy. Archived from the original, 2009. 